\title{
Survey of management of iatrogenic bleeding following bronchoscopic sampling
}

\author{
To the Editor:
}

We read with interest the recent papers by ABuQhyyas et al. [1] and PathaK et al. [2] as well as the associated editorial by HeRTH [3], which all highlighted the safety issues of bronchoscopy with antithrombotic agents. However, there is also a significant issue with regard to a bronchoscopist's ability to manage bleeding and their level of training. Flexible bronchoscopy as an investigative modality is very safe, with an estimated mortality between 1:1000 and 1:10000 patients [4]. The most feared complication is severe bleeding following sampling, which can potentially lead to asphyxiation and/or exsanguination, although the risk of severe bleeding is extremely low at $0.26 \%$ [4]. However, this rarity means that screening for excess bleeding risk may not always occur and the bronchoscopist may not have the skill set or equipment to deal with an acute severe haemorrhage.

We recently performed an electronic survey of respiratory consultants and specialist registrars (SpRs) listed within the Irish and Welsh thoracic societies regarding bleeding following bronchoscopic sampling. Data were collected over a period of 3 months and anonymised, apart from the respondent's designation.

There were 64 responses in total (46 Irish and 18 Welsh) with 65\% of respondents at consultant grade. The estimated frequency of significant bleeding during bronchoscopy with endobronchial sampling, graded as frequently $(10 \%)$, occasionally $(1 \%)$ and rarely $(0.1 \%)$, was $3 \%, 58 \%$ and $39 \%$, respectively. 32 (50\%) performed coagulation tests routinely prior to endobronchial sampling with $23(36 \%)$ only if the patient was on anticoagulation; nine (14\%) would not routinely check coagulation. $42(66 \%)$ stated that they would routinely check coagulation prior to a transbronchial lung biopsy.

Clopidogrel was routinely discontinued by 59 (92\%) of those surveyed prior to endobronchial sampling by a mean (range) of 6.7 (2-14) days. In 63 (98\%), novel oral anticoagulants would be discontinued for a mean of $3.4(1-10)$ days. 62 (97\%) would discontinue warfarin for a mean period of 4.7 (1-7) days prior to endobronchial sampling. 22 (34\%) of respondents would discontinue low-dose aspirin a mean of 5 (1-10) days pre-procedure.

$40(63 \%)$ conducted a safety timeout prior to every procedure whilst $21(33 \%)$ would not, with three $(4 \%)$ not sure if it was performed by the nurse. $41(64 \%)$ had adrenaline and $4^{\circ} \mathrm{C} 0.9 \%$ saline prepared prior to a procedure. Methods employed to control bleeding are shown in figure 1.

Responses regarding the dosage of endobronchial adrenaline employed showed a varied knowledge level: $17(26 \%)$ did not know what dosage of adrenaline could be used initially to control bleeding and $27(43 \%)$ were unaware of the maximum dosage of adrenaline that could be used. 28 (44\%) stated they had access to a balloon tamponade device, 17 (26\%) had no access and $19(30 \%)$ did not know. 63 (98\%) stated they did not practice the use of a balloon tamponade procedure and $43(67 \%)$ would like to receive training in its usage.

$36(56 \%)$ felt confident or very confident in managing a significant bleed, with $16(25 \%)$ somewhat confident and $12(19 \%)$ not confident at all. Preferred methods of up-skilling in the management of acute severe bleeding in 58 responses were: high-fidelity manikin, 36 (62\%); e-learning modules, 29 (50\%); formal local bronchoscopy course, 17 (29\%); virtual reality-based simulation training, 22 (38\%); and SpR study days, 17 (29\%).

@ERSpublications

This survey has revealed significant deficits in anticipating those at risk of severe bleeding from bronchoscopy http://ow.ly/MUoH30icT5X

Cite this article as: Scott A, McDonnell MJ, Ahmed M, et al. Survey of management of iatrogenic bleeding following bronchoscopic sampling. Eur Respir Rev 2018; 27: 170128 [https://doi.org/10.1183/ 16000617.0128-2017]. 


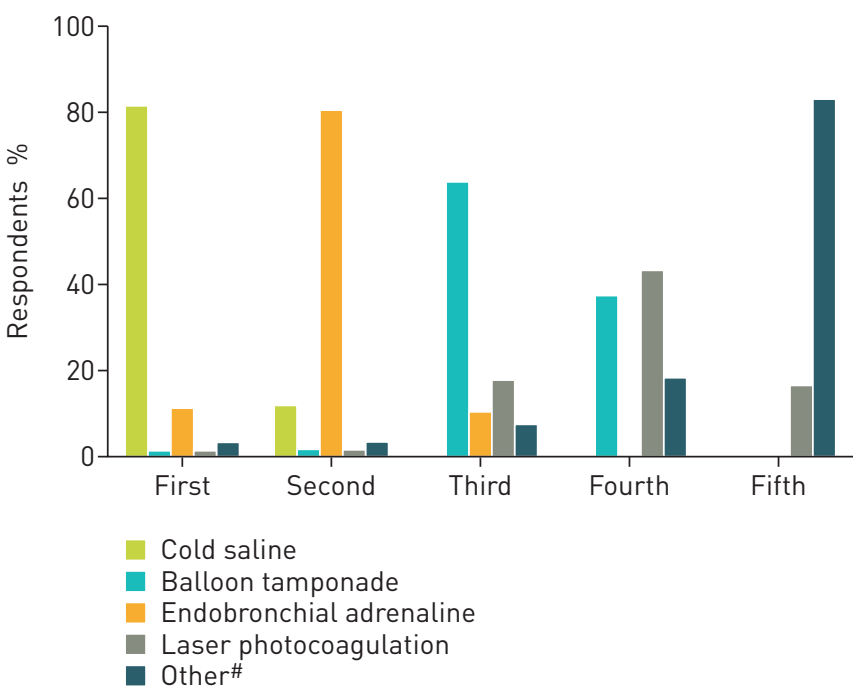

FIGURE 1 Order of methods used to control significant bleeding. " : other methods included wedging the bronchoscope in the bleeding segment ( $n=4)$, argon photocoagulation ( $n=2)$ and an infusion of tranexamic acid ( $n=1)$.

Although the incidence of severe bleeding is rare in bronchoscopic practice, it is still a feared complication. Our survey confirms that severe bleeding is indeed rare, with $96 \%$ of respondents encountering bleeding in $\leqslant 1 \%$ of procedures. $86 \%$ of respondents stated they would check coagulation studies routinely or if on prior anticoagulation before endobronchial sampling. The 2013 British Thoracic Society (BTS) guidelines suggest coagulation studies should be performed in patients with potentially abnormal coagulation, which would only apply to a small number of patients, and that routine checking in low-risk patients is unnecessary. Whether coagulation status should be checked prior to transbronchial biopsy is unclear. Even though there is a tripling of the risk of severe bleeding with this procedure, the prevalence is still $<1 \%$. The present authors' practice is to check coagulation routinely prior to this mode of biopsy. The articles published by ABUQAYYAS et al. [1] and PATHAK et al. [2] provide an excellent review of the current evidence base in the management of antithrombotic agents prior to bronchoscopy, and will help guide daily practice.

Safe discontinuation of antithrombotic agents pre-procedure is an important issue. Our survey has shown errors regarding the safe discontinuation of clopidogrel, which increases the risk of severe bleeding $(27 \%$ versus $0.3 \%$ in controls) when performing transbronchial biopsy [5]. The BTS guidelines also recommend a safety pause with a checklist pre-bronchoscopy but, according to the present survey, this has not been widely adopted. Interestingly, two-thirds of patients with acute severe bleeding have no elevated risk factors, in that bleeding can be largely unpredictable; hence, the correct management of acute bleeding is essential. As per guidelines, the vasoconstrictors cold $0.9 \%$ saline $(82 \%)$ and adrenaline (11\%) were the preferred first choice to achieve haemostasis, with a reversal of these percentages as second-line treatment. Cold saline needs to be pre-prepared but this is not done by a third of respondents, necessitating the use of adrenaline. A significant knowledge deficit was also exposed with regards to the safe use of adrenaline in terms of the correct concentration, dilution and recommended maximum dosage, which should not exceed $1 \mathrm{mg}$ (10 $\mathrm{mL}$ of 1:10000 adrenaline).

Wedging the bronchoscope into the bleeding segment is advised as the third choice by the BTS guidelines but emerges as a fifth choice from this survey. Balloon tamponade devices have a role in bleeding that continues despite vasoconstrictor therapy. Over half of those surveyed stated that they did not have or did not know whether they have a balloon device in their institution, and a large unmet training need was identified regarding this procedure.

Nearly a half of those surveyed were less than confident in managing acute bleeding and nearly all stated that would like further training in managing acute severe bleeding.

This survey has revealed significant deficits in anticipating those at risk of severe bleeding from bronchoscopy, and its subsequent management. The main learning focus should be on awareness of antithrombotic agents and their safe discontinuation, pre-procedure time-out, training in local vasoconstrictor therapy and how to wedge the bronchoscope, and education and training in the usage of a balloon tamponade device. 
Andrew Scott, Melissa J. McDonnell, Mohammed Ahmed, Antoinette Flannery, Irfan Mujammil, David Breen and Robert M. Rutherford

Respiratory Dept, Galway University Hospitals, Galway, Ireland.

Correspondence: Andrew Scott, Respiratory Dept, University Hospital Galway, Newcastle Rd, Galway, H91 YR71, Ireland. E-mail: andrew.scott@hse.ie

Received: Nov 282017 | Accepted: Dec 192017

Conflict of interest: None declared.

Provenance: Submitted article, peer reviewed.

\section{References}

1 Abuqayyas S, Raju S, Bartholomew JR, et al. Management of antithrombotic agents in patients undergoing flexible bronchoscopy. Eur Respir Rev 2017; 26: 170001.

2 Pathak V, Allender JE, Grant MW. Management of anticoagulant and antiplatelet therapy in patients undergoing interventional pulmonary procedures. Eur Respir Rev 2017; 26: 170020.

3 Herth FJF. Bronchoscopy and bleeding risk. Eur Respir Rev 2017; 26: 170052.

4 Du Rand IA, Blaikley J, Booton R, et al. British Thoracic Society guideline for diagnostic flexible bronchoscopy in adults: accredited by NICE. Thorax 2013; 68: Suppl. 1, i1-i44.

5 Ernst A, Eberhardt R, Wahidi $M$, et al. Effect of routine clopidogrel use on bleeding complications after transbronchial biopsy in humans. Chest 2006; 129: 734-737. 